\title{
3 Research Square

\section{Role of the stearyl-coenzyme A desaturase 1 gene in regulating palmitic acid tolerance of goose primary hepatocytes}

\section{Bincheng Tang}

Sichuan Agricultural University

Jia min Qiu

Sichuan Agricultural University

Shen qiang $\mathrm{HU}$

Sichuan Agricultural University

\section{Liang Li}

Sichuan Agricultural University

Ji wen Wang ( $\nabla$ wjw2886166@163.com )

Farm Animal Genetic Resources Exploration and Innovation Key Laboratory, Sichuan Agricultural University, Chengdu, Sichuan

\section{Research}

Keywords: SCD1, goose primary hepatocytes, LO2 cells, palmitic acid tolerance

Posted Date: March 23rd, 2020

DOl: https://doi.org/10.21203/rs.3.rs-18680/v1

License: (c) (i) This work is licensed under a Creative Commons Attribution 4.0 International License. Read Full License 


\section{Abstract}

\section{Background}

Unlike mammals, goose fatty liver shows a strong tolerance to fatty acids without obvious injury. Stearylcoenzyme A desaturase 1 (SCD1) serves crucial role in desaturation of saturated fatty acids (SAFs), but its role in the SAFs tolerance of goose hepatocytes has not been reported. This study was conducted to explore the role of $S C D 1$ in regulating palmitic acid tolerance of goose primary hepatocytes.

\section{Methods}

To evaluate the palmitic acid tolerance of cultured hepatocytes, MTT was examined to reflect the effect of palmitic acid on cell viability, and quantitative PCR was used to detect the mRNA expression levels of several genes related to ER stress, inflammation, and apoptosis, and the role of SCD1 in palmitic acid tolerance of goose hepatocytes was explored using RNA interfere.

\section{Results}

Our results indicated that goose hepatocytes exhibited a higher tolerant capacity to palmitic acid than human hepatic cell line (LO2 cells). Furthermore, the mRNA levels of fatty acid desaturation-related genes (SCD1 and FADS2) and fatty acid elongate enzyme-related gene (ELOVL6) were significantly upregulated in goose primary hepatocytes treated with $0.6 \mathrm{mM}$ palmitic acid. However, in cultured LO2 cells, expression of ER stress-related genes (XBP, BIP and ATF6), inflammatory response-related genes (IL-6, IL$1 \beta$ and IFN- $\gamma$ ) and apoptosis-related genes (Bax, BCl-2, Caspase-3 and Caspase-9) was significantly enhanced by the addition of $0.6 \mathrm{mM}$ palmitic acid. Additionally, siRNA-mediated downregulation of SCD1 significantly reduced the palmitic acid tolerance of goose primary hepatocytes under the treatment of 0.6 $\mathrm{mM}$ palmitic acid; meanwhile, the mRNA expression of inflammatory-related genes (IL-6 and $I L-1 \beta)$ and several key genes involved in the PI3K/AKT, Fox01, mTOR and AMPK pathways (AKT1, AKT2, FOXO1 and SIRT1), as well as the protein expression of cytochrome $\mathrm{C}$ and the apoptosis rate were also upregulated.

\section{Conclusion}

In conclusion, our data suggested that $S C D 1$ is involved in enhancing the palmitic acid tolerance of goose primary hepatocytes by regulating inflammation- and apoptosis-related genes expression.

\section{Introduction}

In mammals, excessive fat deposition in hepatocytes causes ER stress, inflammation and apoptosis, consequently leading the non-alcohol fatty liver to exacerbate into fatty hepatitis [1]. Goose (Anser anser), as a descendant of migrant birds, has an excellent capacity to deposit fat in the liver. In poultry production, this capacity is exploited for producing the fatty liver that is 5- to 10-fold larger than normal liver after 2-3 weeks of overfeeding [2,3]. Interestingly, its liver can develop serious steatosis without overt injury [4], showing minimal inflammation and other immune-related responses, which suggests that 
goose has different physiological and metabolic characteristics from mammals. In a previous study, the mRNA levels of tumor necrosis factor alpha ( $T N F a$ ) was significantly downregulated by overfeeding in the goose liver [5]. Moreover, during the formation of goose fatty liver, expression of ER stress marker genes, $78 \mathrm{kDa}$ glucose-regulated protein (Grp78) and X-box binding protein 1 (Xbp 1), was also inhibited [6]. These results demonstrated that goose liver has a strong tolerance to fatty acids so as to deposit a large amount of fat without the occurrence of lesions.

Stearoyl-CoA desaturase (SCD) is recognized as a pivotal enzyme in the biosynthesis of monounsaturated fatty acids (MUFAs) by catalyzing the insertion of the cis double bond at the delta-9 position of stearoyl-CoA (C16:0) and palmitoyl-CoA (C18:0), which are subsequently converted into corresponding MUFAs, respectively [7]. In the past several decades, studies on SCD1 were mainly related to fat synthesis, as SCD1-knocked mice showed reduced TG content, inhibited de novo synthesis of fatty acids, and less hepatic steatosis [8]. Several recent reports supported the notion that the SCD1 is critical for regulating the ratio of SFAs to MUFAs. Janikewicz et al. [9] reported that SCD1 had an effect on ER stress response of pancreatic $\beta$-cells by altering the SFAs to MUFAs ratio. In another study, Iwai et al. [10] found that SCD1 affected apoptosis in mouse proximal tubular cells by regulating the MUFAs/SFAs ratio. Moreover, it was found that in overfeeding-induced goose fatty liver, the MUFAs/SFAs ratio significantly increased while the content of both palmitic acid and stearic acids decreased $[4,11]$. Thus, it can be generalized that SCD1 exerts an important role in regulating MUFAs/SFAs ratio.

In goose, $S C D 1$ is ubiquitously expressed in metabolic tissues and shows higher expression levels in liver $[1,12]$. In addition, goose SCD1 has higher genomic copy number than that of humans and chickens [13]. Therefore, we hypothesized that $S C D 1$ could be involved in the regulation of goose hepatic steatosis. To test our hypothesis, this study aims to 1) compare the effects of palmitic acids at different concentrations on cell viability using MTT as well as expression of genes related to ER stress, inflammation, and apoptosis between goose primary hepatocytes and LO2 cells, 2) examine the effects of downregulating SCD1 expression via RNA interference on cell viability as well as expression of ER stress, inflammation, and apoptosis-related genes in goose hepatocytes under the treatment palmitic acid, and 3) investigate the effects of downregulating SCD1 expression via RNA interference on expression of key genes involvement of PI3K/AKT, Fox01, mTOR and AMPK pathways in goose hepatocytes under the treatment palmitic acid. These data may provide a better understanding of the molecular mechanisms underlying SCD1 regulating the tolerance of SFAs in goose primary hepatocytes.

\section{Materials And Methods}

\section{Isolation and Culture of Goose Primary Hepatocytes}

Hepatocytes were isolated from ten 20-day-old Tianfu meet geese (Anser anser) that were hatched at the same time and raised under the condition of natural light and temperature at the Experimental Farm for Waterfowl Breeding of Sichuan Agricultural University (Sichuan, China) according to the methods introduced by Seglen [14], and were then cultured with Dulbecco's modified Eagle's medium (DMEM; 
Hyclone, Utah, USA) containing $10 \%$ fetal bovine serum (Gibco, USA). The cells were incubated at $40^{\circ} \mathrm{C}$ in a humidified atmosphere containing $5 \% \mathrm{CO}_{2}$, and the medium was renewed after 3 hours of culture. 24 hours later, the medium was replaced with serum-free DMEM medium.

\section{Culture of LO2 Cells}

LO2 cells was purchased from Cell Bank of Chinese Academic of Science (Shanghai, China). Cells were cultured at $37^{\circ} \mathrm{C}$ and $5 \% \mathrm{CO}_{2}$ in Dulbecco's modified Eagle's medium (DMEM; Hyclone, Utah, USA), supplemented with $10 \%$ fetal bovine serum (Gibco, USA). Culture medium was changed every 2 days.

\section{siRNA Transfection}

Cultured goose primary hepatocytes were seeded in 6-well plates and incubated for 24 hours. Cells were transfected with $0.6 \mathrm{mM}$ siRNA interfere SCD1 or negative control siRNA (GenePharma Co.,Ltd, Shanghai, China) using Lipofectamine ${ }^{\circledR} 3000$ transfection reagent (Invitrogen, USA), and incubated for 24 hours with Dulbecco's modified Eagle's medium (DMEM; Hyclone, Utah, USA) containing 10\% fetal bovine serum (Gibco, USA). The siRNA sequences are as follows: siRNA-230, GCG AUA CGU CUG GAG GAA UTT (sense) and AUU CCU CCA GAC GUA UCG CTT (antisense); siRNA-602, GCG ACA UAA AGG CCG ACA A (sense) and UUG UCG GCC UUU AUG UCG C (antisense); siRNA-818, CGU ACG AUC AGA ACA UCA A (sense) and UUG AUG UUC UGA UCG UAC G (antisense).

\section{MTT Assay}

The assay for cell viability was performed to Natali et al. [15]. Goose primary hepatocytes and LO2 cells were plated at a density of $1 \times 10^{4}$ cells/well in a 96-well culture dish. After 24 hours, cells were then incubated for 4 hours with $1 \mathrm{mg} / \mathrm{mL}$ MTT in a $37^{\circ} \mathrm{C}$ incubator, which is converted from the yellow tetrazolium compound to the purple formazan derivative by mitochondria of living cells. After removal of the unconverted MTT, the formazan product was dissolved in DMSO and the formazan dye absorbance was measured at $490 \mathrm{~nm}$.

\section{Oil Red 0 staining}

Cells were stained with Oil-Red-O to examine the amount of fat accumulation in the cells. Goose primary hepatocytes and LO2 cells were cultured on 6-well culture slides, fixed in formalin and stained using a method described by Ramirez-zacarias et al. [16]. Briefly, the wells were fixed with $10 \%$ Neutral Formalin Fix Solution for 1 hour, rinsed with PBS and then stained with Oil-Red-O for 2 hours. After 2 hours, remove free Oil-Red-0, and rinsed with PBS. The Oil-Red-0 was extracted with the addition of $100 \%$ isopropanol and Oil Red-O was determined in aliquots from wells after shaking the culture plates for $20 \mathrm{~min}$ at room temperature.

\section{TUNEL Assay}


TUNEL staining was performed with an insitu apoptosis detection kit (Beyotime Biotechnology, Shanghai, China). TUNEL-positive and -negative cells were counted in four randomly selected areas of a culture slide. The results are expressed as a ratio of TUNEL-positive cells to total cell number in each field.

\section{Cytochrome c Immunofluorescence and DAPI Assay}

The cyt-c protein expression in goose primary hepatocytes was detected by using Cytochrome $\mathrm{c}$ (Cytochrome $\mathrm{C}$ antibody) Kit (Beyotime Biotechnology, Shanghai, China) according to the manufacturer's instruction. DAPI staining was used with DAPI Staining Solution (Beyotime Biotechnology, Shanghai, China), and cells were incubated for $10 \mathrm{~min}$ with $0.5 \mathrm{ug} / \mathrm{mL}$ DAPI Solution in a $37^{\circ} \mathrm{C}$ incubator.

\section{RNA Extraction and cDNA Synthesis}

Total RNA was extracted from cultures cells using TRIzol Reagent (Invitrogen) according to the manufacturer's instruction. The quality and quantity of total RNA were checked by electrophoresis on a $1.5 \%$ agarose gel. The cDNA was obtained by a cDNA Synthesis Kit (Takara, Japan) under the manufacturer's protocol with 1 ug of total RNA as a template.

\section{Quantitative Real-time PCR (qRT-PCR)}

The primers (Table 1) used for qRT-PCR were designed using the Primer Premier 5 software (Premier Biosoft International, USA). GADPH (GenBank NO. MG674174.1) and $\beta$-actin (GenBank NO. M26111.1) were used as the internal controls. The mRNA expression levels of target genes were measured by qRTPCR. The qRT-PCR was performed in a 96-well Bio-Rad iQ5 (Bio-Rad Laboratories, USA) using a Takara ExTaq RT-PCR Kit and SYBR Green as the detection dye (Takara, Japan). Real-time PCR was carried out under the following condition: 1 cycle of pre-denaturation at $95^{\circ} \mathrm{C}$ for 10 seconds; 40 cycles of $95^{\circ} \mathrm{C}$ for 5 seconds, and $60^{\circ} \mathrm{C}$ for 40 seconds, and starting at a temperature of $55^{\circ} \mathrm{C}$ and increasing by $0.5^{\circ} \mathrm{C}$ every 10 seconds to determine primer specificity. All cDNA samples were tested three times, and the results were normalized to the levels of goose GADPH and $\beta$-actin expression.

\section{Data Analysis}

The relative mRNA expression of target gene was calculated by the comparative $\mathrm{Ct}$ method $\left(2^{-\Delta \Delta C t}\right.$ methods) [17]. All data were subjected to analysis of variance (ANOVA), and the means were compared for significance using Tukey's test. The ANOVA and t-tests were performed using the SPSS 23.0 software (IBM, USA). Differences were considered statistically significant at $P<0.05$.

\section{Results}

\section{Comparative analysis of the palmitic acid tolerance of goose and human hepatocytes}

The results showed that goose primary hepatocytes had a higher tolerant capacity to palmitic acid than LO2 cells (Fig. 1). When treated with $0.2-0.6 \mathrm{mM}$ palmitic acid, the cell viability of goose primary 
hepatocytes was not change, but under 0.7-0.9 mM treatment, the cell viability significantly decreased $(P<0.05)$. For LO2 cells, when treated with 0.2-0.9 mM palmitic acid, the cell viability significantly decreased $(P<0.05)$, and with the increase of palmitic acid concentration, the cell viability decreased continuously.

\section{Effects of palmitic acid on the mRNA levels of ER stress-, inflammation- and apoptosis-related genes in goose primary hepatocytes and LO2 cells}

To further verify palmitic acid tolerance of goose primary hepatocytes, we then compared the mRNA levels of ER stress-, inflammation-, and apoptosis-related genes in both cells. As shown in Fig.2, when treated with $0.6 \mathrm{mM}$ palmitic acid, the mRNA levels of ER stress-related genes (XBP, BIP and ATF6), inflammatory response-related genes (IL-6, IL-1 $\beta$ and IFN- $\gamma$ ) and apoptosis-related genes (Bax, Bcl-2, Caspase-3 and Caspase-9) were not significantly different from the control group in goose primary hepatocytes, but in the LO2 cells their expression levels significantly increased compared to the control group $(P<0.05)$.

\section{Effects of palmitic acid on lipid accumulation and involved gene expression in goose primary hepatocytes and LO2 cells}

As shown in Fig.3, under the treatment of $0.6 \mathrm{mM}$ palmitic acid, the lipid accumulation of goose primary hepatocytes was significant higher than LO2 cells $(P<0.05)$. As shown in Fig. 4, the mRNA levels of fatty acid desaturation-related genes (SCD1and FADS2) was significantly promoted $(P<0.05)$ in goose primary hepatocytes, while no significant differences were seen in LO2 cells. In addition, the mRNA expression of the fatty acid elongate enzyme-related gene (ELOVL6) in both cells significantly increased $(P<0.05)$, whereas that of triglyceride synthesis-related gene (DGAT2) remained statically unchanged in these two cells.

\section{Effects of SCD1 downregulation on cell viability and the mRNA levels of ER stress-, inflammation- and apoptosis-related genes in goose primary hepatocytes}

The expression profile of $S C D 1$ promoted us to investigate its function in palmitic acid tolerance of goose primary hepatocytes. Then, we interfered $S C D 1$ in goose primary hepatocytes by using siRNA. As shown in Fig.5, siRNA-818 was the most effective silencing $S C D 1(P<0.05)$. Therefore, subsequent experiments used siRNA-818 for $S C D 1$ interference. Under the treatment of $0.6 \mathrm{mM}$ palmitic acid, downregulation of $S C D 1$ significantly reduced the tolerance of goose primary hepatocytes to palmitic acid $(P<0.05)$. As shown in Fig.6, under the treatment of $0.6 \mathrm{mM}$ palmitic acid, downregulation of $S C D 1$ significantly increased the mRNA levels of inflammation related genes $(I L-6$ and $I L-1 \beta)$, the protein expression of cytochrome $\mathrm{C}$ and the apoptosis rate. However, genes involved in ER stress (XBP, BIP and ATF6), inflammatory (IFN- $\gamma$ ) and apoptosis (Bax, Bcl-2, Caspase 3 and Caspase 9) were not significantly changed under the treatment. 


\section{Effects of SCD1 downregulation on the mRNA levels of PI3K/AKT, Fox01, mTOR and AMPK pathway- related genes in goose primary hepatocytes}

Finally, we detected the expression of $S C D 1$ regulatory pathway-related genes after downregulating $S C D 1$. Under the treatment of $0.6 \mathrm{mM}$ palmitic acid, downregulation of $S C D 1$ in goose primary hepatocytes significantly increased the mRNA expression of pathway key genes (AKT1, AKT2, FOXO1 and SIRT1), and the expression of $P I 3 K$ was decreased $(P<0.05)$, while that of $m T O R$ and $A M P K$ were not no significantly altered (Fig.7).

\section{Discussion}

Palmitic acid is a major type of SFAs in the liver and plays essential roles in maintaining liver health. Previous study reported that $0.2 \mathrm{mM}$ palmitic acid markedly decreased the cell viability in HepG2 cells [18]; in contrast, our laboratory demonstrated that the cell viability of goose primary hepatocytes was not changed by treatment with $0.6 \mathrm{mM}$ palmitic acid [19], suggesting that there may be significant differences in the palmitic acid tolerance between goose and human hepatocytes. In this study, the palmitic acid of 0.2-0.9 mM was used to treat goose primary hepatocytes and LO2 cells cultured in vitro. Our results showed that the maximum tolerant concentration to palmitic acid of goose primary hepatocytes was $0.6 \mathrm{mM}$, which was consistent with the results of Pan et al. [19]; however, the maximum tolerance concentration of palmitic acid in LO2 cells is less than $0.2 \mathrm{mM}$, which was consistent with the results of the study on HepG2 cells [18]. The above results showed that goose primary hepatocytes have a higher tolerance to palmitic acid than LO2 cells, which may be one of the reasons why goose liver has an excellent capacity for fat accumulation.

To fully explore palmitic acid tolerance of goose primary hepatocytes, we then compared the mRNA levels of ER stress-, inflammation-, and apoptosis- related genes in goose primary hepatocytes and LO2 cells. ER stress, inflammation, and apoptosis are important basis for evaluating the tolerance of fatty acids in hepatocytes. ER stress occurs extensively in the livers of individuals with steatohepatitis [20]. Previous studies indicated that addition of palmitic acid increased the expression of ER stress-related genes in mammalian hepatocytes [21, 22]. In this study, we found that treatment with $0.6 \mathrm{mM}$ palmitic acid had no significant effect on the mRNA expression of ER stress-related genes (XBP, BIP and ATF6) in goose primary hepatocytes. However, in LO2 cells, levels of ER stress-related genes were markedly increased $(P<0.05)$, and mRNA levels of BIP was increased more than 10 -fold. XBP, ATF6 and BIP are located on three pathways that cause the UPR response of ER stress [20], the mRNA expression of the three genes did not change significantly in goose primary hepatocytes, indicating that goose primary hepatocytes have a strong tolerance to palmitic acid in ER stress response. In addition, increasing evidence indicated that treatment of mammalian hepatocytes with palmitic acid can increase the content of cellular proinflammatory factors $[23,24]$. Inflammatory factors $I L-6, I L-1$, and $I F N-y$ are used as biomarkers of hepatitis in mammals, and their expression has significantly increased in mammalian fatty hepatitis [25]. Both ER stress and inflammatory response lead to massive apoptosis of cells. Our results showed that addition of palmitic acid had no significant effect on the mRNA levels of the inflammatory response- 
related genes (IL-6, IL-1 $\beta$ and IFN- $\gamma$ ) and apoptosis-related genes (Bax, BCl-2, Caspase-3 and Caspase-9) in goose primary hepatocytes, but in LO2 cells their mRNA levels markedly increased compared to the control group $(P<0.05)$. Remarkably, the mRNA levels of Bax were increased more than 3 -fold, which is consistent with the results in HepG2 cells [26]. Therefore, we conclude that goose hepatocytes were more tolerant to palmitic acid than LO2 cells in ER stress, inflammation and apoptosis.

Results from previous studies reveled that overfeeding significantly increased expression of genes related to fat synthesis in the goose liver $[13,27]$. In the liver, palmitic acid elongation the carbon chain is mainly catalyzed by ELOVL6 enzyme, and desaturation under the action of SCD1, FADS1, and FADS2, and finally forms non-toxic triglycerides under the action of DGAT2 [28]. And the above five genes mRNA levels significantly increased in the goose liver after overfeeding [13]. In our study, the levels of fatty acid desaturation-related genes (SCD1 and FADS2) mRNA was markedly enhanced by addition of $0.6 \mathrm{mM}$ of palmitic acid $(P<0.05)$ in goose primary hepatocytes, while no significant differences were seen in LO2 cells. We also found that the expression of the fatty acid elongate enzyme-related gene (ELOVL6) in both cells significantly increased $(P<0.05)$, whereas that of triglyceride synthesis-related gene $(D G A T 2)$ remained statically unchanged in these two cells. Together, these results indicated that palmitic acid has an important effect on the fatty acid desaturation process in goose hepatocytes, and also indicates that the fatty acid desaturation process may play an important role to palmitic acid tolerance of goose liver.

SCD1 is the key regulatory enzyme responsible for the desaturation of SFAs, which can desaturation palmitic acid into MUFAs. Moreover, increasing studies have shown that the mRNA levels of SCD1 was increased in overfeeding-induced goose fatty liver $[1,12,13]$. Therefore, this study was to study the effects of SCD1 on palmitic acid tolerance of goose primary hepatocytes. In our study, under the treatment of $0.6 \mathrm{mM}$ palmitic acid, downregulation of $S C D 1$ was able to decrease the tolerance of goose primary hepatocytes to palmitic acid $(P<0.05)$, indicating that $S C D 1$ play crucial role in palmitic acid tolerance of goose hepatocytes. Then, we further investigated the palmitic acid tolerance of goose primary hepatocytes in ER stress, inflammatory and apoptosis after downregulating with SCD1. Our data showed that downregulation of SCD1 had no significant effect on the mRNA expression of ER stressrelated genes (XBP, BIP and ATF6) 区indicating that SCD1 did not affect the palmitic acid tolerance of goose primary hepatocytes in ER stress response. Furthermore, $S C D 1$ downregulation resulted in the mRNA levels of inflammatory factors $(I L-6$ and $I L-1 \beta)$ were significantly increased, suggesting that $S C D 1$ can regulate palmitic acid tolerance of goose primary hepatocytes in inflammation pathway where $/ L-6$ and $I L-1 \beta$ are located. Moreover, our results showed that downregulation of $S C D 1$, the mRNA levels of apoptosis related genes ( $\mathrm{Bax}, \mathrm{BCl}-2$, Caspase 3 and Caspase 9) were not significantly changed, but the protein expression of cytochrome $C$ and the apoptosis rate significantly increased, which indicated that $S C D 1$ increases palmitic acid tolerance goose primary hepatocytes by inhibiting apoptosis. These data demonstrated that $S C D 1$ plays a critical role in mediating palmitic acid tolerance of goose primary hepatocytes through inflammation and apoptosis response.

Finally, to further explore the specific pathways of $S C D 1$ regulating palmitic acid tolerance of goose primary hepatocytes. Then, we tested the mRNA levels of key genes in the SCD1 regulatory pathways. In 
our study, we found that under the treatment of $0.6 \mathrm{mM}$ palmitic acid, downregulation of SCD1 significantly increased the mRNA expression of AKT1, AKT2, FOXO1 and SIRT1, while that of $m T O R$ and $A M P K$ were not significantly altered, indicating $S C D 1$ can regulate the AKT/FoxO1 pathway at the transcription level. In addition, SIRT 1 can regulate FoxO1 through acetylation, and early studies have demonstrated that FoxO1 play an important role in regulating inflammation and apoptosis [29-31], but the regulatory relationship between SCD1 and SIRT1 has not been reported. Therefore, we speculated that SCD1 may regulate the AKT/Fox01 pathway through SIRT1/Fox01 pathway or increase the ratio of SFAs/MUFAs to inhibit the occurrence of inflammation and apoptosis response. Together, these data support a conclusion that $S C D 1$ may increase the palmitic acid tolerance of goose primary hepatocytes by regulating the AKT/Fox01, SIRT1/FoxO1 pathways to inhibit the occurrence of inflammatory and apoptosis.

In summary, data from the present study suggested that goose primary hepatocytes have a higher tolerance to palmitic acid than LO2 cells and that SCD1 has a crucial role in enhancing the palmitic acid tolerance of goose primary hepatocytes by regulating inflammation- and apoptosis-related genes expression.

\section{Declarations}

\section{Availability of data and materials}

The datasets used and/or analyzed during the current study are available from the corresponding author on reasonable request.

\section{Acknowledgments}

We thank all participants at our laboratory for their ongoing assistance.

\section{Funding}

This work was supported by the China Agricultural Research System (CARS-42-4), and the Project of National Science and Technology Plan for the Rural Development in China (2015BAD03B06).

\section{Author information}

Bincheng Tang and Jiamin Qiu contributed equally to this work.

\section{Affiliations}

Farm Animal Genetic Resources Exploration and Innovation Key Laboratory, Sichuan Agricultural University, Chengdu, Sichuan, 61130, P.R. China

Bincheng Tang, Jiamin Qiu, Shenqiang Hu, Liang Li, and Jiwen Wang 


\section{Author's contributions}

Deigned and directed the study: BCT, JMQ, JWW. Performed the experiments: BCT, JMQ. Analyzed the data: BCT, JMQ, SQH. Wrote the manuscript: BCT. Reviewed the manuscript: LL, SQH. All authors approved the final version of this manuscript.

\section{Corresponding author}

Correspondence to Jiwen Wang

\section{Ethics declarations}

\section{Ethics Statement and consent to participate}

All experimental protocols involving animal manipulation were approved by the Institutional Animal Care and Use Committee of Sichuan Agricultural University (Ya'an, Sichuan, China) under the approved number: DKY-B201000805. Written informed consent was obtained from all participants.

\section{Consent for publication}

All authors signed the informed consent.

\section{Competing interests}

The authors declare that they have no competing interests.

\section{Abbreviations}

SCD1: Stearyl-coenzyme A desaturase 1

SAFs: saturated fatty acids

LO2 cells: human hepatic cell line

TNFa: tumor necrosis factor alpha

Grp78: 78 kDa glucose-regulated protein

Xbp1: X-box binding protein 1

MUFAs: monounsaturated fatty acids

\section{References}

1. Zhang R, Zhu L, Zhang Y, Shao D, Wang L, Gong D. cDNA cloning and the response to overfeeding in the expression of stearoyl-CoA desaturase 1 gene in Landes goose. Gene. 2013;512(2):464-469. 
2. Hermier D, Rousselot-Pailley D, Peresson R, Sellier N. Influence of orotic acid and estrogen on hepatic lipid storage and secretion in the goose susceptible to liver steatosis. Bba-Lipid Lpid Met. 1994;1211(1):97-106.

3. Mourot J, Guy G, Peiniau P, Hermier D. Effects of overfeeding on lipid synthesis, transport and storage in two breeds of geese differing in their capacity for fatty liver production. Anim Res. 2006;55(5):427-442.

4. Osman RH, Liu L, Xia L, Zhao X, Wang Q, Sun X, Zhang Y, Yang B, Zhang Y, Gong D, Geng T. Fads1 and 2 are promoted to meet instant need for long-chain polyunsaturated fatty acids in goose fatty liver. Mol Cell Biochem. 2016;418(1-2):103-117.

5. Geng T, Yang B, Li F, Xia L, Wang Q, Zhao X, Gong D. Identification of protective components that prevent the exacerbation of goose fatty liver: characterization, expression and regulation of adiponectin receptors. Comp Biochem Phys B. 2016;194:32-38.

6. Geng T, Xia L, Li F, Xia J, Zhang Y, Wang Q, Yang B, Montgomery S, Cui H, Gong D. The role of endoplasmic reticulum stress and insulin resistance in the occurrence of goose fatty liver. Biochem Bioph Res Co. 2015;465(1):83-87.

7. Hodson L, Fielding BA. Stearoyl-CoA desaturase: rogue or innocent bystander?. Prog Lipid Res. 2013;52(1):15-42.

8. Miyazaki M, Flowers MT, Sampath H, Chu K, Otzelberger C, Liu X, Ntambi JM. Hepatic stearoyl-CoA desaturase-1 deficiency protects mice from carbohydrate-induced adiposity and hepatic steatosis. Cell Metab. 2007;6(6):484-496.

9. Janikiewicz J, Hanzelka K, Dziewulska A, Kozinski K, Dobrzyn P, Bernas T, Dobrzyn A. Inhibition of SCD1 impairs palmitate-derived autophagy at the step of autophagosome-lysosome fusion in pancreatic $\beta$-cells. J Lipid Res. 2015;56(10):1901-1911.

10. Iwai T, Kume S, Chin-Kanasaki M, Kuwagata S, Araki H, Takeda N, Sugaya T, Uzu T, Maegawa H, Araki SI. Stearoyl-CoA desaturase-1 protects cells against lipotoxicity-mediated apoptosis in proximal tubular cells. Int J Mol Sci. 2016;17(11):1868.

11. Molette C, Berzaghi P, Zotte AD, Remignon H, Babile R. The use of near-infrared reflectance spectroscopy in the prediction of the chemical composition of goose fatty liver. Poultry Sci. 2001;80(11):1625-1629.

12. Pan Z, Han C, Wang J, Li L, Tang H, Lv J, Lu L, Xu F. Cloning and expression of stearoyl-CoA desaturase 1 (SCD-1) in the liver of the Sichuan white goose and landes goose responding to overfeeding. Mol Bio Rep. 2011;38(5):3417-3425.

13. Lu L, Chen Y, Wang Z, Li X, Chen W, Tao Z, Shen J, Tian Y, Wang D, Li G, Chen L, Chen F, Fang D, Yu L, Sun Y, Ma Y, Li J, Wang J. The goose genome sequence leads to insights into the evolution of waterfowl and susceptibility to fatty liver. Genome Bio. 2015;16(1):89.

14. Seglen PO. Preparation of rat liver cells: III. Enzymatic requirements for tissue dispersion. Exp Cell Res. $1973 ; 82(2): 391-398$. 
15. Natali F, Siculella L, Salvati S, Gnoni GV. Oleic acid is a potent inhibitor of fatty acid and cholesterol synthesis in C6 glioma cells. J Lipid Res. 2007;48(9):1966-1975.

16. Ramirez-Zacarias JL, Castro-Munozledo F, Kuri-Harcuch W. Quantitation of adipose conversion and triglycerides by staining intracytoplasmic lipids with Oil red O. Histochemistry. 1992;97(6):493-497.

17. Livak KJ, Schmittgen TD. Analysis of relative gene expression data using real-time quantitative PCR and the 2- $\Delta \Delta$ CT method. Methods. 2001;25(4):402-408.

18. Ji J, Zhang L, Wang P, Mu Y, Zhu X, Wu Y, Yu H, Zhang B, Chen S, Sun Z. Saturated free fatty acid, palmitic acid, induces apoptosis in fetal hepatocytes in culture. Exp Toxicol Pathol. 2005;56(6):369376.

19. Pan Z, Wang J, Tang H, Li L, Lv J, Xia L, Han C, Xu F, He H, Xu H, Kang B. Effects of palmitic acid on lipid metabolism homeostasis and apoptosis in goose primary hepatocytes. Mol Cell biochem. 2011;350(1-2):39-46.

20. Fu S, Watkins SM, Hotamisligil GS. The role of endoplasmic reticulum in hepatic lipid homeostasis and stress signaling. Cell Metab. 2012;15(5):623-634.

21. Wang D, Wei Y, Pagliassotti MJ. Saturated fatty acids promote endoplasmic reticulum stress and liver injury in rats with hepatic steatosis. Endocrinology. 2006;147(2):943-951.

22. Kakisaka K, Cazanave SC, Fingas CD, Guicciardi ME, Bronk SF, Werneburg NW, Mott JL, Gores GJ. Mechanisms of lysophosphatidylcholine-induced hepatocyte lipoapoptosis. Am J Physiol-Gastr L. 2012;302(1):G77-G84.

23. Joshi-Barve S, Barve SS, Amancherla K, Gobejishvili L, Hill D, Cave M, Hote P, McClain, CJ. Palmitic acid induces production of proinflammatory cytokine interleukin-8 from hepatocytes. Hepatology. 2007;46(3):823-830.

24. Miura K, Yang L, van Rooijen N, Brenner DA, Ohnishi H, Seki E. Toll-like receptor 2 and palmitic acid cooperatively contribute to the development of nonalcoholic steatohepatitis through inflammasome activation in mice. Hepatology; 2013;57(2):577-589.

25. Paschos P, Paletas K. Non alcoholic fatty liver disease and metabolic syndrome. Hippokratia. 2009;13(1):9.

26. Malhi H, Bronk SF, Werneburg NW, Gores GJ. Free fatty acids induce JNK-dependent hepatocyte lipoapoptosis. J Biol Chem. 2006;281(17):12093-12101.

27. Zhu L, Meng H, Duan X, Xu G, Zhang J, Gong D. Gene expression profile in the liver tissue of geese after overfeeding. Poultry science. 2011;90(1):107-117.

28. Ntambi JM (Ed.). Hepatic de novo lipogenesis and regulation of metabolism. Springer. 2015.

29. Brown J, Wang H, Suttles J, Graves DT, Martin M. Mammalian target of rapamycin complex 2 (mTORC2) negatively regulates Toll-like receptor 4-mediated inflammatory response via Fox01. J Biol Chem. 2011;286(52):44295-44305.

30. Tan S, Shui G, Zhou J, Shi Y, Huang J, Xia D, Wenk MR, Shen H. Critical role of SCD1 in autophagy regulation via lipogenesis and lipid rafts-coupled AKT-FOXO1 signaling pathway. Autophagy. 
2014;10(2):226-242.

31. Chung S, Lee TJ, Reader BF, Kim JY, Lee YG, Park GY, Karpurapu M, Ballinger MN, Qian F, Rusu L, Chung HY, Unterman TG, Croce CM, Christman JW. FoxO1 regulates allergic asthmatic inflammation through regulating polarization of the macrophage inflammatory phenotype. Oncotarget. 2016;7(14):17532.

Figures

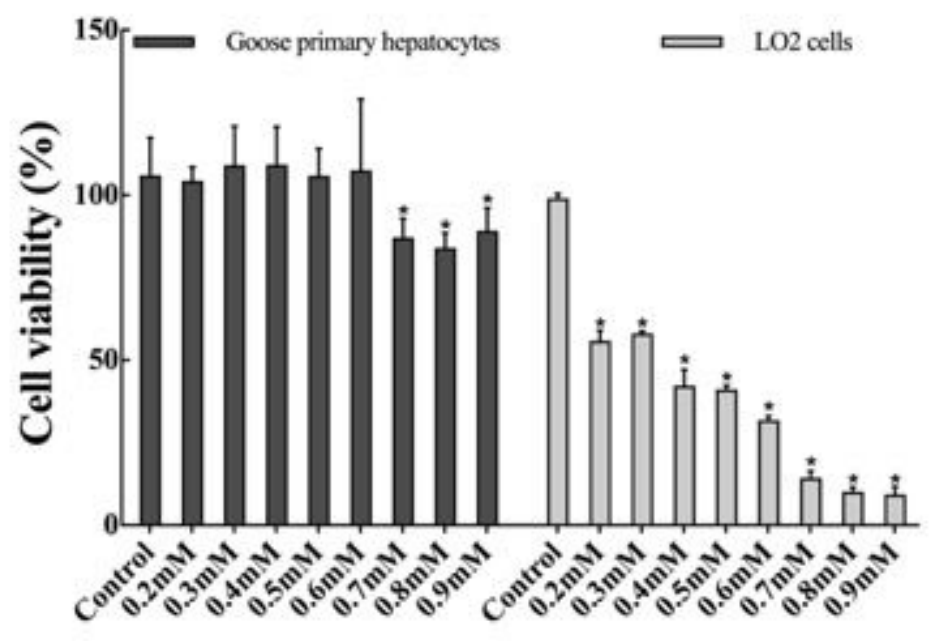

Figure 1

Effects of palmitic acid at different concentrations on cell viability of goose primary hepatocytes and L02 cells. * indicates a significant difference at $\mathrm{P}<0.05$ compared to the control group.
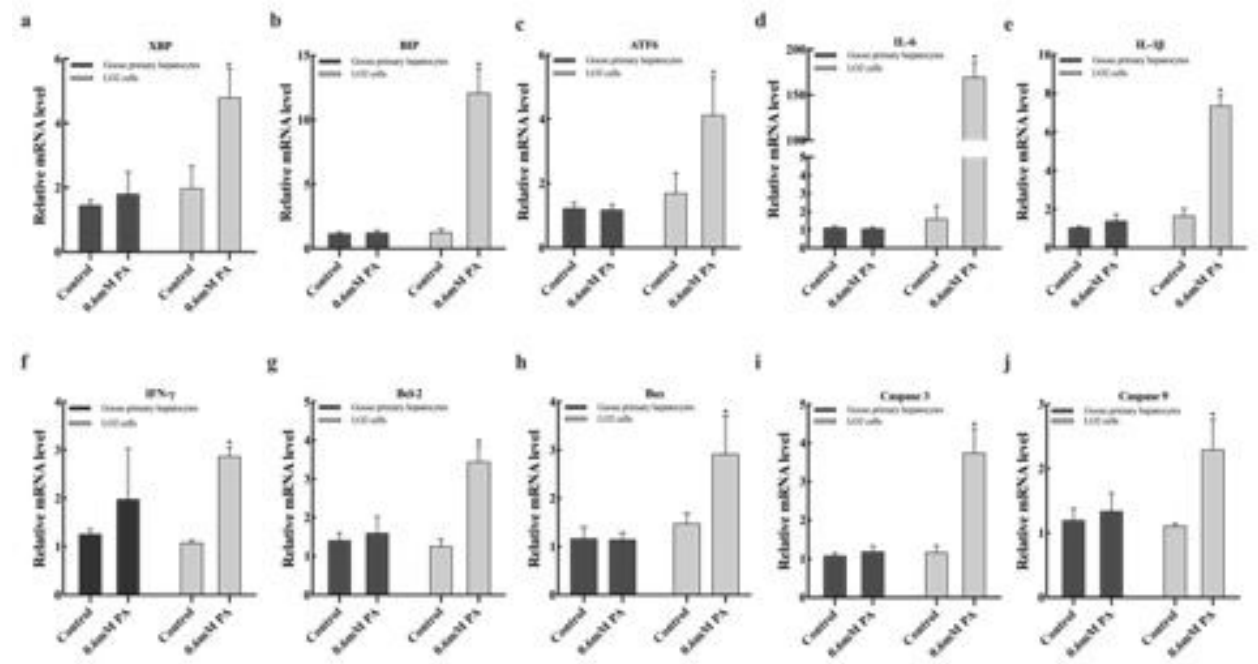

Figure 2

(a-c) Effects of palmitic acid on ER stress-related genes mRNA expression in goose primary hepatocytes and LO2 cells; (d-f) Effects of palmitic acid on inflammation-related genes mRNA expression in goose primary hepatocytes and LO2 cells; (g-j) Effects of palmitic acid on apoptosis-related genes mRNA 
expression in goose primary hepatocytes and LO2 cells. * indicates a significant difference at $\mathrm{P}<0.05$ compared to control group of the same species.
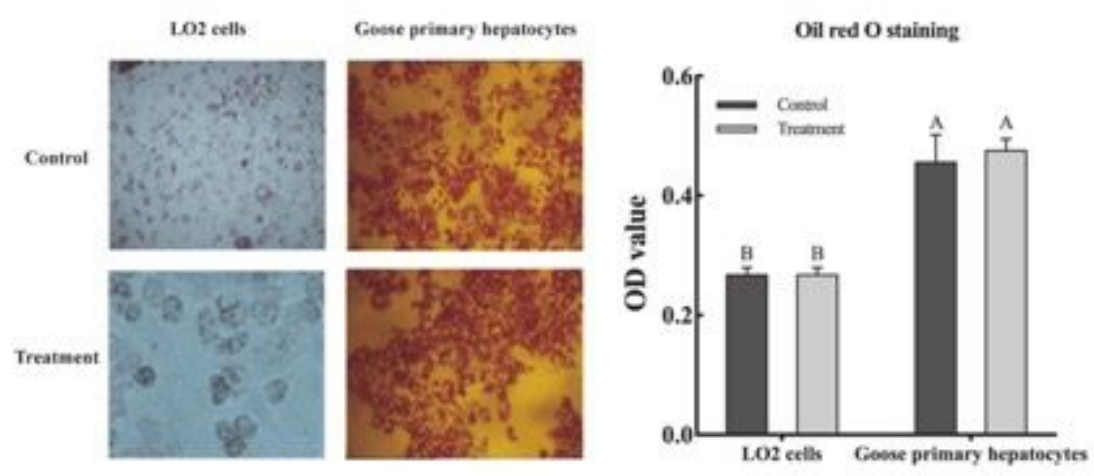

\section{Figure 3}

Effects of palmitic acid on lipid accumulation in goose primary hepatocytes and LO2 cells. Different uppercase letters indicated a significant difference $(P<0.05)$.
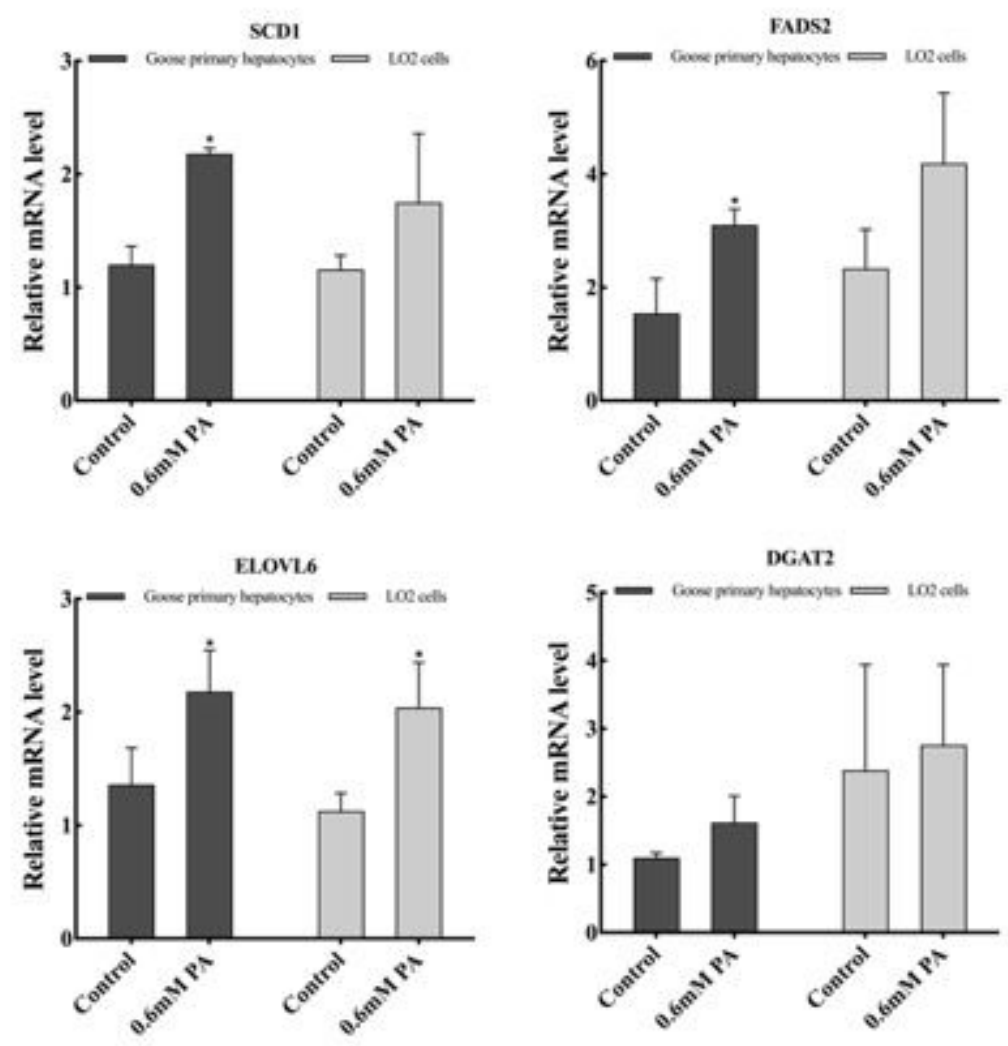

Figure 4

Effects of palmitic acid on TG synthesis related-genes expression in goose primary hepatocytes and LO2 cells. * indicates a significant difference at $\mathrm{P}<0.05$. 

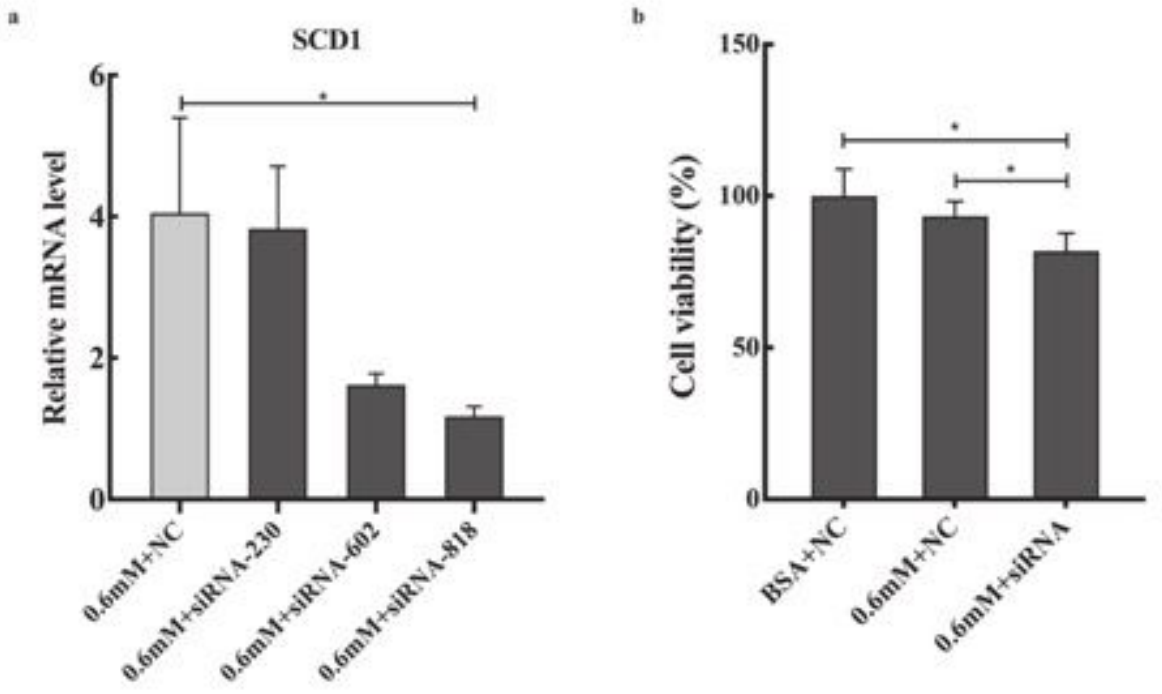

Figure 5

(a) The different siRNA silencing efficiency; (b) Effects of SCD1 downregulation on goose primary hepatocytes cell viability under palmitic acid treatment. * indicates a significant difference at $P<0.05$.
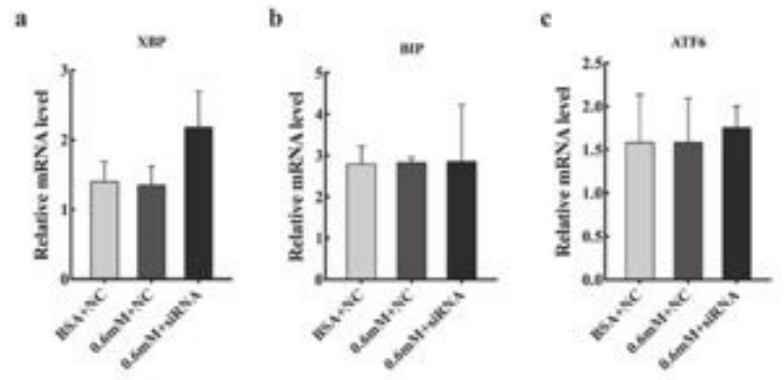

d
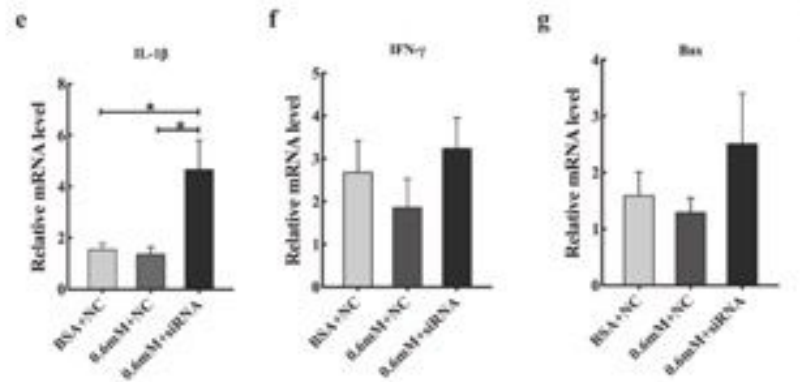

h
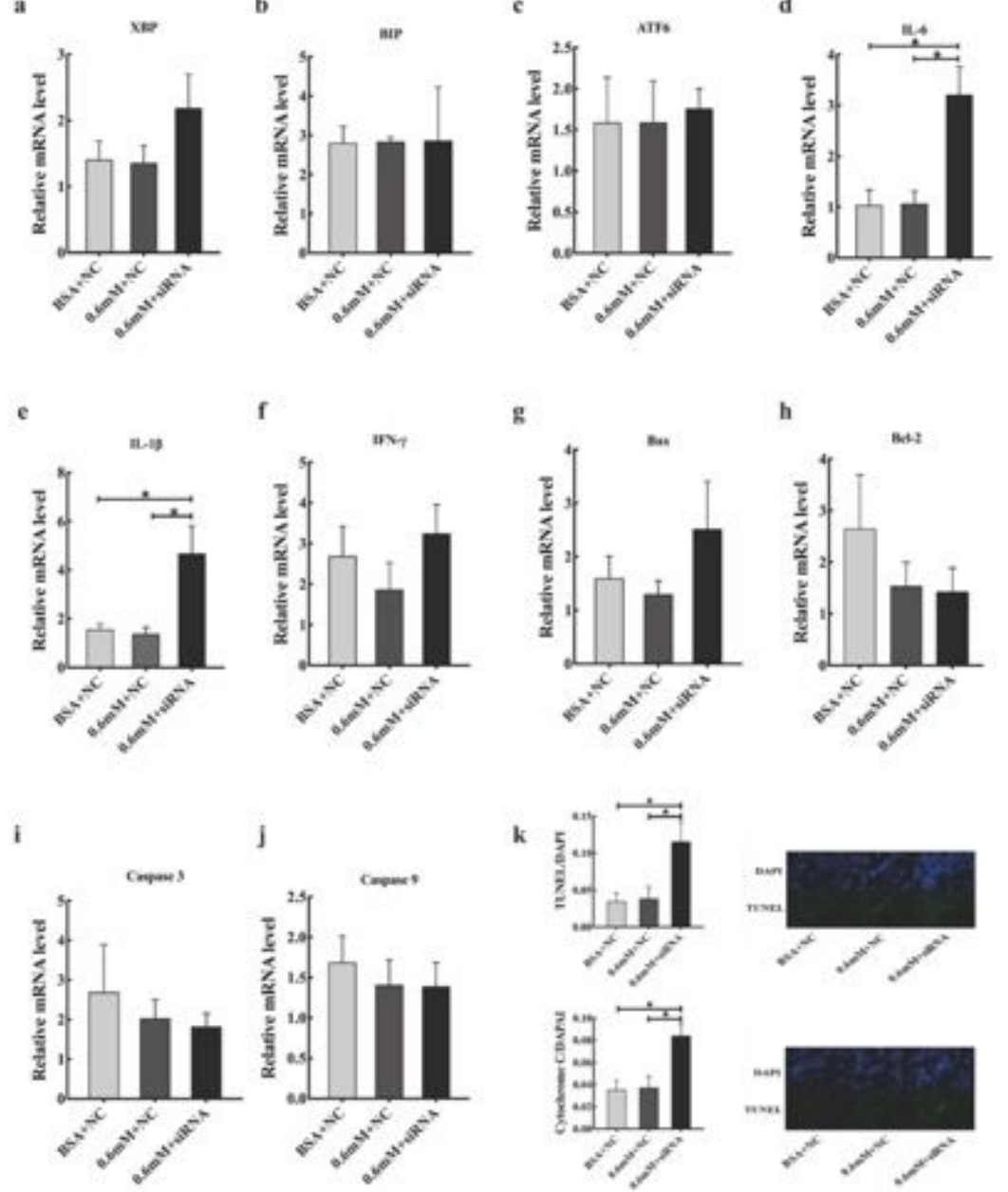

Figure 6 
(a-c) Effects of SCD1 downregulation on the mRNA expression of ER stress-related genes in goose primary hepatocytes under palmitic acid treatment; (d-f) Effects of SCD1 downregulation on the mRNA expression of inflammation-related genes in goose primary hepatocytes under palmitic acid treatment; ( $\mathrm{g}$ i) Effects of SCD1 downregulation on the mRNA expression of apoptosis-related genes in goose primary hepatocytes under palmitic acid treatment; (j) Effects of SCD1 downregulation on the apoptosis rate and cyt-c protein expression in goose primary hepatocytes under palmitic acid treatment. * indicates a significant difference at $\mathrm{P}<0.05$.
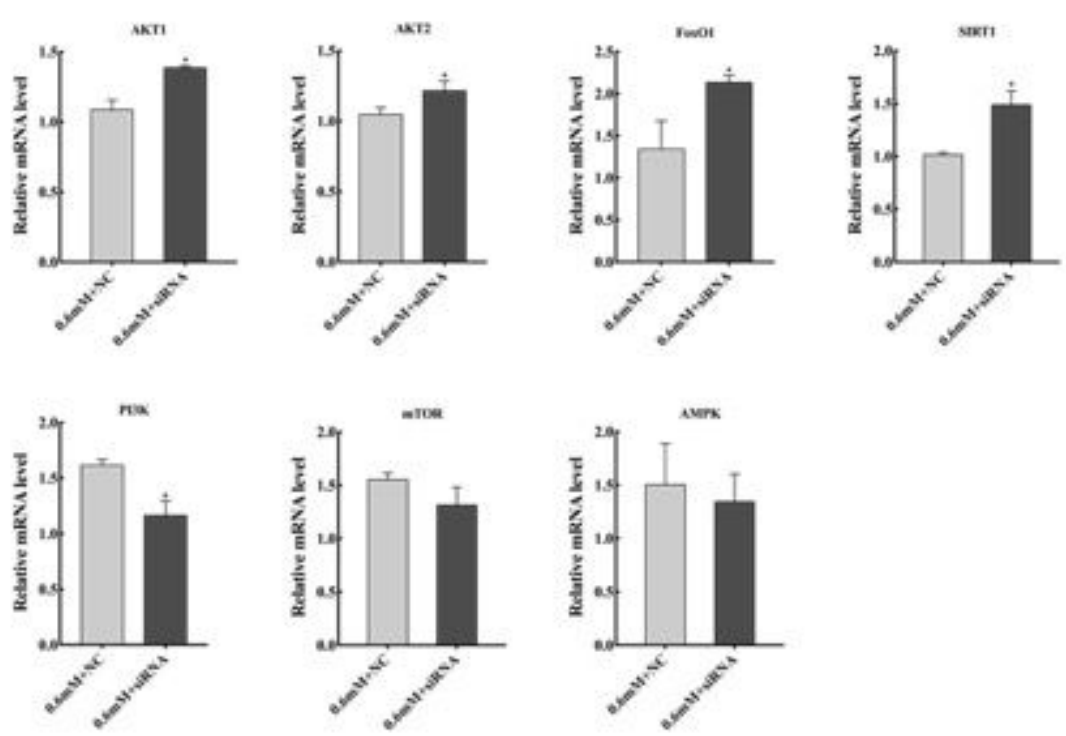

Figure 7

Effects of SCD1 downregulation on the mRNA levels of PI3K/AKT, Fox01, mTOR and AMPK pathwayrelated genes in goose primary hepatocytes under palmitic acid treatment. * indicates a significant difference at $P<0.05$. 